\title{
Agent Technology Applied to Adaptive Relay Setting for Multi-Terminal Lines
}

\author{
D. V. Coury \\ Member IEEE \\ Dept of Electrical Eng. \\ Escola de Engenharia de São Carlos \\ University of São Paulo \\ Brazil
}

\author{
J. S. Thorp \\ Fellow IEEE \\ School of Electrical Engineering \\ Cornell University \\ Ithaca, NY 14853 \\ USA
}

\author{
K. M. Hopkinson \\ Student Member IEEE \\ Department of Computer Science \\ Cornell University \\ Ithaca, NY 14853 \\ USA
}

\begin{abstract}
This paper discusses the adaptation of the settings of distance relays for multi-terminal lines employing agents. Agents are software processes capable of searching for information in networks, interacting with pieces of equipment and performing tasks on behalf of their owners (relays). Results illustrating the performance of the adaptive method proposed compared to conventional fixed settings are presented. It is shown that the digital relays and agents acting within a communication structure (also called middleware) can alter adaptive settings to ensure correct performance over a wide variety of operation conditions, without the need of an additional communication link. The proposed relaying scheme can also be utilized for first zone clearing over the entire line.
\end{abstract}

Keywords: Distance Protection, Relaying, Three-terminal Lines, Agent Technology.

\section{I.INTRODUCTION}

Transmission lines may be tapped to provide intermediate connections to loads, or to reinforce the underlying lower voltage network through a transformer and such a configuration is well known as a multi-terminal line [1].

Protection of multi-terminal lines is not as simple as that of two-terminal lines. They usually experience additional problems caused by the intermediate infeed from the third terminal, an outfeed, different line lengths to the tee point, etc. The limitations of non-pilot distance relays for their protection have been acknowledged for decades. These limitations lead to the traditional distance relaying approach to experience severe underreach and overreach problems. The relay first zone reach may not even extend beyond the tee point in some underreach cases [2].

Some approaches based on adaptive relaying were proposed to face such a situation and some fundamental concepts can be found in [3]. Horowitz et al [4] define adaptive relaying as a protection philosophy which permits and seeks to make adjustments to various protection functions in order to make them more attuned to prevailing power system conditions. An adaptive setting concept for two and three terminal lines, which can respond to changes in the network conditions, was proposed by Xia et al $[5,6]$.

Stedall et al [7] also proposed an investigation into the use of adaptive setting techniques for improved distance back-up protection.

This paper discusses the adaptation of the settings of distance relays for multi-terminal lines employing agents. Agents are software processes capable of searching for information in networks, interacting with pieces of equipment and performing tasks on behalf of their owners (relays). Moreover, they are autonomous and cooperative. Very few publications concerning the application of agents in the protection field have been reported in the literature. Tomita et al [8] proposed a cooperative protection system utilizing agents. Relay agents were constructed, and the cooperation of the main agents was simulated for primary, backup and adaptive protection.

Results illustrating the improved performance of the adaptive method proposed compared to conventional fixed settings are presented in the following sections.

\section{II.AGENT TECHNOLOGY}

A software agent is a computer program that takes independent action based on events in the surrounding environment [9]. Under control of some application that owns the agent, it is sent forth or placed at some site within a network where it takes local actions on behalf of its owners. Agents are dynamic entities. For example, an agent can exhibit dynamically changing behavior, receiving updated parameter settings from the owner or from other agents participating in the same application. An agent can also learn from the environment; the term "intelligent" is sometimes applied to such agents, particularly if the learning method involves artificial intelligence tools, or neural networks.

Agents can be further subdivided based on their mobility. Mobile agents can literally travel from one location to another while maintaining their state and functionality. One imagines such an agent as moving through a network in search of certain data, or for locations having certain attributes. In contrast, local agents are placed by the owner and then remain in the same location throughout their computational lives. Both kinds of agents normally have the ability to communicate with one another making mobility a lesser benefit than it might appear at first glance.

In the present paper, we consider the simplest kinds of agents: local, non-intelligent ones. Our work seeks to demonstrate advantages stemming from the rapid response time made possible by placing a computational task close to the point where an action or measurement is needed, and the simplicity of the power systems architecture afforded by the ability to send code to general purpose computing platforms. The basic idea is that by doing so, new control paradigms can be introduced and executed efficiently, without changing 
the basic computing architecture of the power systems network.

The use of agents raises security considerations that go beyond those encountered in traditional software systems. Among the issues that need to be considered are the mechanism for installing agents and for communicating parameter updates to them, the means by which a computing platform or agent authenticates incoming messages, and the enforcement of agent resource restrictions, such as the use of scarce computational cycles or space in databases and file systems. Prior to deploying agents in mission-critical settings, one would need satisfying answers on all of these fronts. Preliminary work towards policies addressing these issues can be found in [11].

\section{III.THE PROPOSED COMMUNICATION STRUCTURE AND THE POWER SYSTEM STUDIED}

\section{A. The communication structure (middleware)}

The computing infrastructure in which our agent community will operate is a subject of active speculation at the time of this writing. The existing power grid communication infrastructure is composed of a hodgepodge of equipment ranging from high-end fiber optic cables with large data bandwidth to crude systems with limited data transfer capabilities to nothing at all. Few utility communications lines are interconnected. However, this picture will change as utilities deploy ever more elaborate control mechanisms, demanding increasingly rich communication frameworks, steps that can easily be foreseen as part of the battle to improve operating efficiencies.

The relay scheme proposed here has modest network resource requirements compared to what we believe will be available in the near future. It is expected that the need for greater communication will result in a Utility Intranet (UI) that would interconnect control areas and their dependent entities using a relatively high capacity network similar to the Internet but physically separate from it and dedicated to the power systems application. However, we caution that predictions of network bandwidth requirements and total network capacity can be deceptively reassuring. History has shown a tendency for networked applications to use all bandwidth made available to them.

To evaluate the feasibility of employing agents and network communication in power-systems relay protection, we need to understand the reliability and latency requirements of the relays. By doing so, we can determine whether or not a proposed combination of network hardware and communication protocols can meet the protection system's needs. Relays play a critical role in power grid operations with low tolerance for failures. One can identify that situations in communication between agents used for relay control might need to take place in as little as $10 \mathrm{~ms}$, a latency realm leaving little time to recover from data transmission failures. It is therefore critical to ask whether available technology can meet those requirements.

The future Utility Intranet will certainly be built from offthe-shelf Internet-based technology. To do otherwise would be unreasonably costly both in terms of initial construction costs and the concomitantly large maintenance requirements. Unfortunately, Internet routing hardware lacks any notion of service quality in network transmissions. Routers, as currently designed, drop packets when load rises and do so even before congestion occurs. This feature is intended to give the TCP (Transmission Control Protocol) a chance to reduce data rates before a router becomes completely overloaded and begins to drop packets. The approach makes sense for web browsers and email systems, but we need to ask what implication this behavior will have for power systems control. Within the Internet, all data delivery is executed on a "best-effort" basis without any possibility to raise the priority of one message over any other. In control applications, there are often situations where one needs to generate high priority events, and we should ask about the implications of running such applications over a network that lacks the corresponding capability.

These points suggest that power systems may need guarantees of "Quality of Service" (QoS). Unfortunately, while QoS has been a very active research topic for many years, there is such deep disagreement over the right approach to take (stemming from cost implications associated with most proposals) that no single solution has gained market acceptance, nor are standard solutions likely in the near future.

In order to face some of the network limitations, communication protocols like Cornell's Spinglass [12] have been developed. Spinglass allows the user to achieve high levels of reliability using protocols that probabilistically mask latency fluctuations and message loss. Spinglass could be optimized for use in power relaying and might form a powerful infrastructure for improved protection schemes. Underlying the study reported here is the idea that if we can simply understand the real needs of agent-based relay protection algorithms, we can identify areas of mismatch between these algorithms and the Internet and can then turn to Spinglass as a source of possible mechanisms to overcome the problematic network behavior.

\section{B. The three-ended system utilized}

In order to simulate the real system encountered in practice, this work makes use of a digital simulator of faulted transmission lines known as PSCAD/EMTDC (Electromagnetic Transients and Controls Simulation Engine)[13]. It should be mentioned that although the technique described is based on Computer Aided Design (CAD), practical considerations such as the Capacitor Voltage Transformer (CVT), Current Transformer (CT) and 


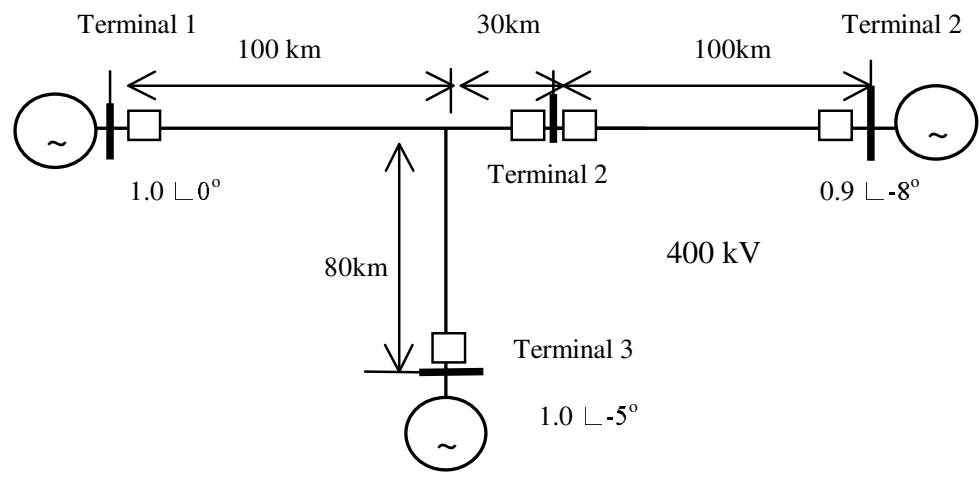

Fig. 1 The three-ended system utilized for tests

anti-aliasing filters on system fault data were also included in the simulation. A $400 \mathrm{kV}$ system with tee configuration is shown in Fig. 1. The different line lengths are presented in the figure, as well as the voltage levels at the busbars. This work focuses on line-to-ground faults, since most faults in power system transmission lines are of this type.

The protection system associated with this configuration was simulated for conventional non-pilot protection as well as a portion of the agent based relaying approach proposed. Digital distance relays with three zone quadrilateral characteristics located at terminals 1,2 and 3 were simulated. DFT (Discrete Fourier Transform) routines were utilized in order to filter the faulted waves. Circuit breakers were associated with the relays in order to isolate the line in case of an internal fault.

\section{IV.AGENT RELAYS}

Fig.2 presents the agent mechanism for protection purposes. It can be initially divided into an adaptive pre-fault approach and a post-fault transfer trip action. The mechanism utilizes local agents at terminals 1,2 and 3 communicating with each other.

\section{A. Adaptive Pre-fault Approach}

The main purpose of the proposed agent mechanism is to carry out the adaptation of the settings of distance relays for multi-terminal lines in order to ensure correct performance over a wide variety of operation conditions.

Initially, local agents for terminals 1,2 and 3 can be defined as:

(1) Operation Agent: This is a local agent, which monitors the operating condition at a terminal. The following parameters related to the system condition must be obtained from on-line measurements and/or calculations:

- Pre-fault busbar voltages

- Power transfer angles

- Source impedances
(2) Breaker Status Agent: This local agent monitors changes in the system topology based on circuit breaker status.

The two local agents, shown in Fig.2, must communicate with the Coordination Agent, as defined in item 3.

(3) Coordination Agent: The coordination agent collects information, makes decision, and disseminates information to the other two Coordination agents. The Coordination Agent at each terminal can then choose the correct relay characteristic, based on the information received.

We propose that a group of settings be calculated off-line, and that these parameters be renewed from time to time as the system condition changes beyond a certain limit, based on the Coordination Agent information. Then, when a fault occurs the relay will operate close to its ideal characteristic.

\section{B. First Zone Transfer Trip}

This action takes place after the fault occurrence. In order to decrease the clearance time, when a fault is detected in the first zone of any of the relays located at the three terminals, a trip signal is sent through the communication network to the other two ends. A First Zone Local Agent coordinates the first zone transfer trip.

The substation host computer executes the communication between local agents at different terminals, as described in the earlier section. It also receives relevant information in order to adapt local relays to the best characteristic available for the system operating condition. The new setting is then downloaded to the relay.

\section{Hierarchical Structure}

The hierarchical system for distance protection, based on agent technology, is shown in Fig. 3. Digital relays are the lowest level of this hierarchy. All the digital relays are linked to the substation host computer. This host acts as a data concentrator for all historical and oscillography records collected by the digital relays. It also has an interface to the station operators [14]. The substation host computer is finally connected to a communication network, as described in section III.A. 


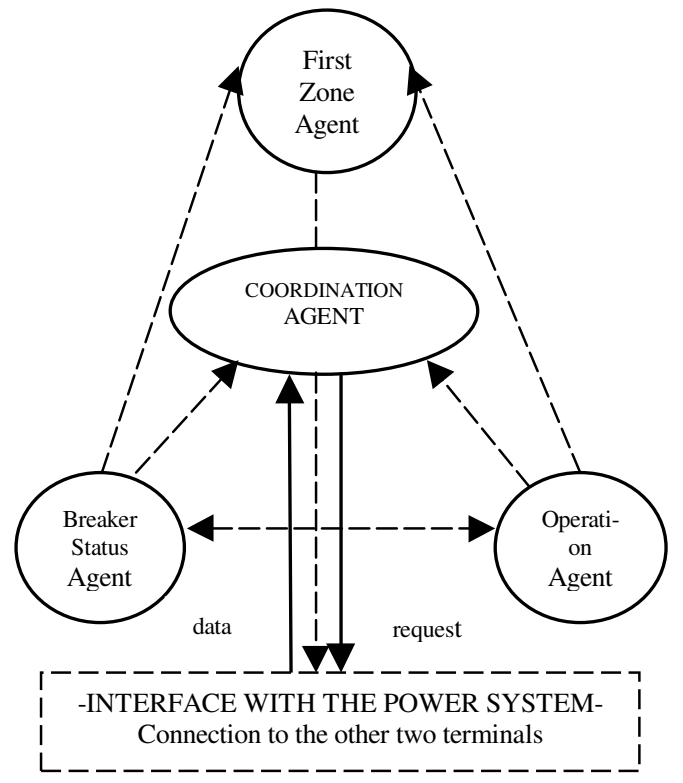

Fig. 2 Agent mechanism in one terminal for protection purpose

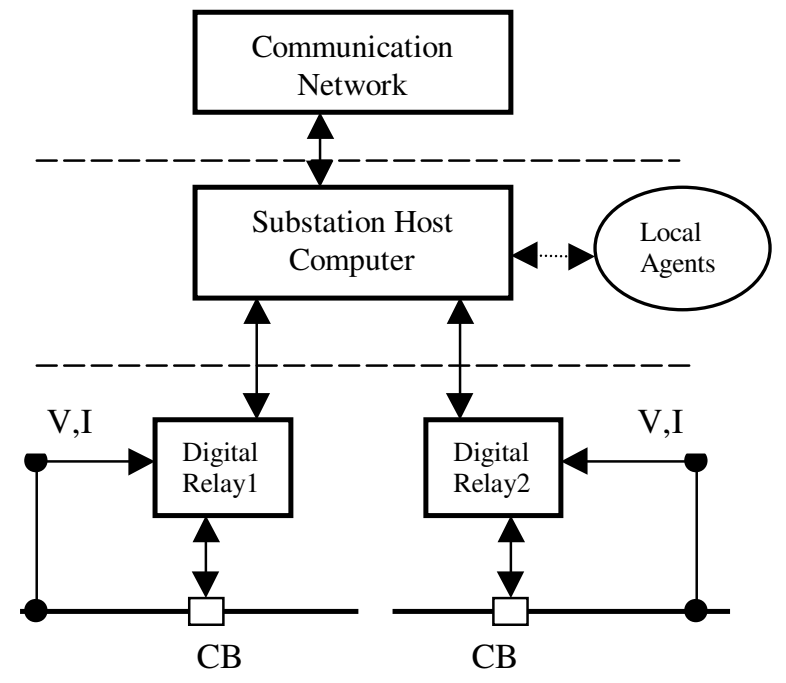

Fig. 3 Hierarchical System

\section{Discussion}

One issue that has not been raised up to this point is why agents are used in the proposed protection system rather than another means of signal processing and communication. We expect that a number of new applications will be developed to take advantage of the Utility Intranet's potential. It is likely that new feature requirements will arise that were never anticipated. A flexible UI communication architecture based on open standards is the best insurance against a never-ending series of incompatible system updates. The integrated computation and communication, that are the defining characteristics of agent technology, form an excellent foundation for a general approach to power systems solutions utilizing a Utility Intranet. When one wishes to add intelligence or other characteristics to an agent operation, it is easily done within the agent framework. Similarly, new communication protocols or application-level additions such as data stream compression can be implemented in agents without affecting the other portions of agent operations. New tasks can be added seamlessly by adding new agents to a system rather than modifying existing code. Mobility is also an easy extension to agent operation, if it is required. In short, agents were designed to operate at a level of abstraction that is both appropriate and compatible with a dynamic system like the developing utility communications infrastructure when coupled with the expected growth of applications that take advantage of its potential.

\section{RESULTS OBTAINED UTILIZING AGENT TECHNOLOGY - AN IMPROVEMENT}

\section{A. Adaptive Pre-fault Approach}

Distance relaying techniques have attracted considerable attention for the protection of transmission lines. The principle of this technique measures the impedance at a fundamental frequency between the relay location and the fault point, thus determining if a fault is internal or external to a protection zone. The relationship between voltage and current is used for this purpose. This relationship is disturbed when there is infeed or outfeed. As mentioned earlier, this can make the relay experience severe underreach or overreach problems, especially considering non-pilot protection schemes for three terminal lines. Another situation that has to be taken into account is when the tap is not working for some reason. The setting of distance relays must reflect all the situations described earlier. Fig. 4(a) shows the typical non-pilot distance protection with different zones for a three terminal line at the condition represented in Fig. 1. As shown in the figure, only approximately 20 from $210 \mathrm{~km}$ obtained instantaneous trip from the three ends of the lines in the case of an internal fault. Fig. 4(b) illustrates the improvement that can be found with the adaptive scheme utilizing Agents that is proposed in this work. The instantaneous trip area increased to approximately $67 \mathrm{~km}$ (more than three times the non-pilot condition) for the same situation. As mentioned before, the proposed adaptive scheme can alter settings to ensure better performance over a wide variety of operation conditions.

\section{B. First Zone Transfer Trip}

In order to highlight the efficiency of the proposed scheme, this section presents some tables comparing the clearance time for the conventional non-pilot protection as well as for the proposed scheme utilizing Agents.

Table 1 presents the clearance time for the three-ended line shown in Fig. 1, considering a non-pilot protection. Line-to-ground faults are applied to different locations on leg 1-T, considering different fault inception angles and fault resistance of $10 \mathrm{ohms}$. 


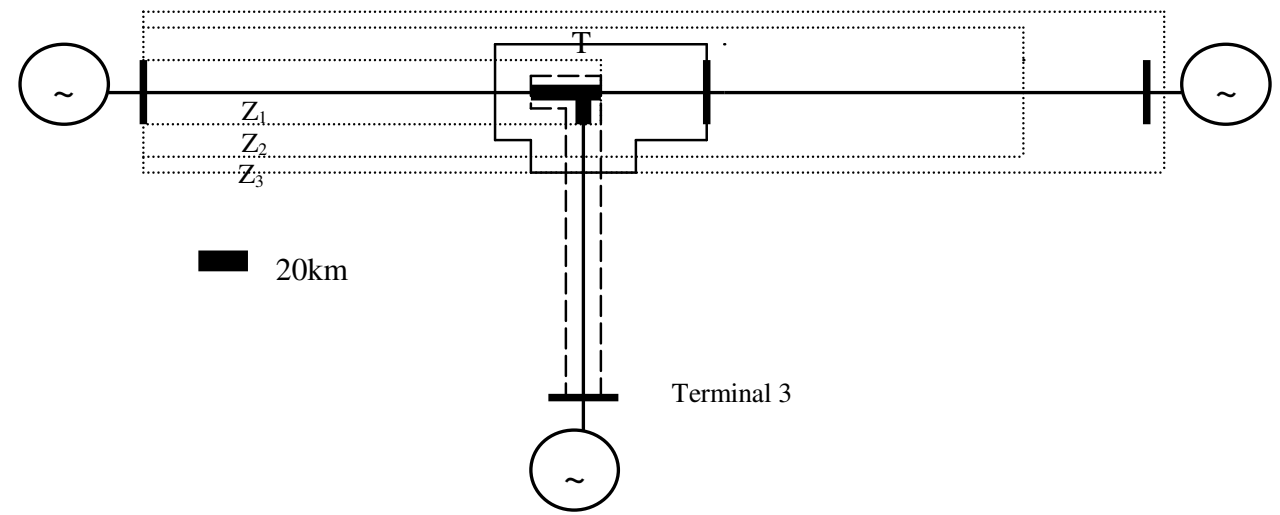

(a) Non-pilot protection

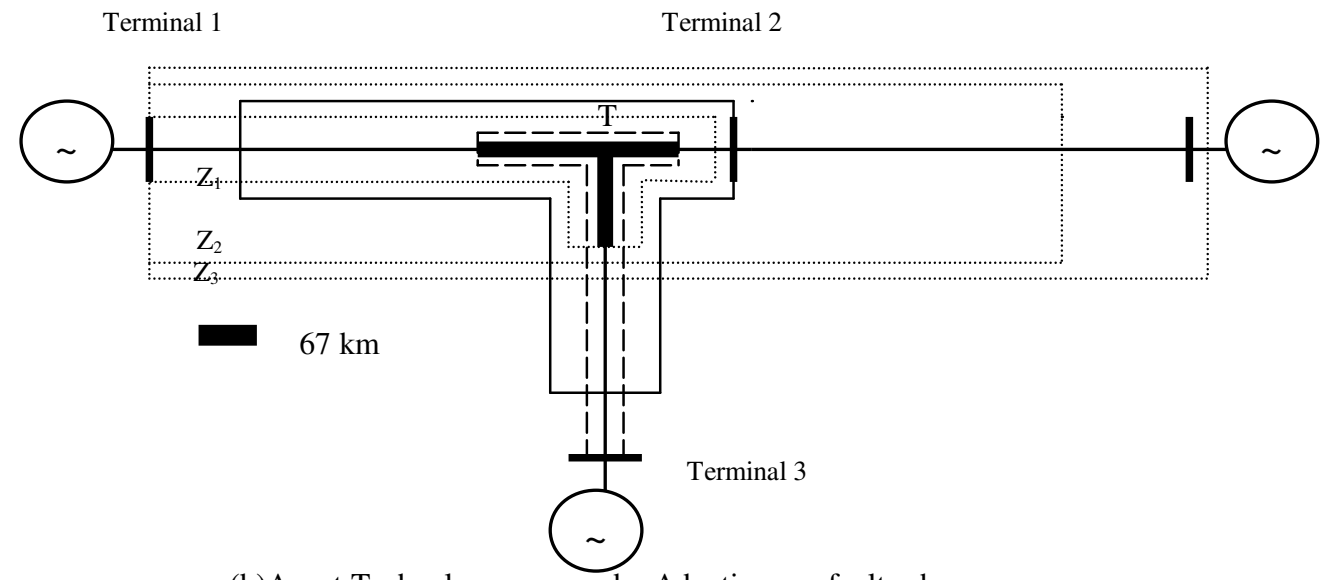

(b)Agent Technology proposed - Adaptive pre-fault scheme

Fig. 4 Distance relaying of a three ended transmission line

Table 1 Clearance time for a three-ended line non-pilot protection, considering different fault locations on leg 1-T $(100 \mathrm{~km})$, different fault inception angles, line-to-ground fault and $\mathrm{Rf}=$ 10 ohms.

\begin{tabular}{|c|c|c|c|c|c|c|c|}
\hline \multirow{2}{*}{$\begin{array}{c}\text { Dist. } \\
\text { from } \\
1 \\
(\mathbf{k m})\end{array}$} & \multirow{2}{*}{$\begin{array}{c}\text { Incep } \\
\text { angle } \\
\text { (o) }\end{array}$} & \multicolumn{2}{|c|}{ Terminal 1} & \multicolumn{2}{|c|}{ Terminal 2} & \multicolumn{2}{|c|}{ Terminal 3} \\
\hline & & $\begin{array}{l}\text { Z } \\
\text { o } \\
\text { n } \\
\text { e }\end{array}$ & $\begin{array}{l}\text { Clea- } \\
\text { rance } \\
\text { Time } \\
\text { (sec.) }\end{array}$ & $\begin{array}{l}\mathbf{Z} \\
\mathbf{o} \\
\mathbf{n} \\
\mathbf{e}\end{array}$ & $\begin{array}{l}\text { Clea- } \\
\text { rance } \\
\text { Time } \\
\text { (sec.) }\end{array}$ & $\begin{array}{l}\text { Z } \\
\text { o } \\
\text { n } \\
\text { e }\end{array}$ & $\begin{array}{l}\text { Clea- } \\
\text { rance } \\
\text { Time } \\
\text { (sec.) }\end{array}$ \\
\hline 10 & $0^{\circ}$ & $\mathrm{Z1}$ & 0.053 & $\mathrm{Z2}$ & 0.359 & $\mathrm{Z} 2$ & 0.354 \\
\hline 20 & $0^{\circ}$ & $\mathrm{Z1}$ & 0.053 & $\mathrm{Z2}$ & 0.359 & $\mathrm{Z} 2$ & 0.354 \\
\hline 30 & $0^{\circ}$ & $\mathrm{Z1}$ & 0.053 & $\mathrm{Z} 2$ & 0.359 & $\mathrm{Z} 2$ & 0.354 \\
\hline 40 & $0^{\circ}$ & $\mathrm{Z1}$ & 0.053 & $\mathrm{Z} 2$ & 0.359 & $\mathrm{Z} 2$ & 0.354 \\
\hline 50 & $90^{\circ}$ & $\mathrm{Z1}$ & 0.047 & $\mathrm{Z} 2$ & 0.353 & $\mathrm{Z} 2$ & 0.353 \\
\hline 60 & $90^{\circ}$ & $\mathrm{Z1}$ & 0.048 & $\mathrm{Z2}$ & 0.355 & $\mathrm{Z} 2$ & 0.350 \\
\hline 70 & $90^{\circ}$ & $\mathrm{Z1}$ & 0.048 & $\mathrm{Z2}$ & 0.355 & $\mathrm{Z} 2$ & 0.348 \\
\hline 80 & $90^{\circ}$ & $\mathrm{Z1}$ & 0.053 & $\mathrm{Z1}$ & 0.057 & $\mathrm{Z} 2$ & 0.354 \\
\hline 90 & $45^{\circ}$ & $\mathrm{Z1}$ & 0.051 & $\mathrm{Z1}$ & 0.056 & $\mathrm{Z1}$ & 0.055 \\
\hline 100 & $45^{\circ}$ & $\mathrm{Z} 1$ & 0.051 & $\mathrm{Z1}$ & 0.056 & $\mathrm{Z} 1$ & 0.054 \\
\hline $\begin{array}{c}\text { aver- } \\
\text { age }\end{array}$ & & & 0.051 & & 0.2668 & & 0.293 \\
\hline
\end{tabular}

Table 2 Clearance time for a three-ended line using Agent theory, considering different fault locations on leg 1-T $(100 \mathrm{~km})$, different fault inception angles, line-to-ground fault and $\mathrm{Rf}=$ $10 \mathrm{ohms}$. Notice the dramatic improvements in protection zones relative to Table 1 .

\begin{tabular}{|c|c|c|c|c|c|c|c|}
\hline \multirow{2}{*}{$\begin{array}{l}\text { Dist. } \\
\text { from } \\
1 \\
(\mathbf{k m})\end{array}$} & \multirow{2}{*}{$\begin{array}{l}\text { Incep } \\
\text { angle } \\
\text { (o) }\end{array}$} & \multicolumn{2}{|c|}{ Terminal 1} & \multicolumn{2}{|c|}{ Terminal 2} & \multicolumn{2}{|c|}{ Terminal 3} \\
\hline & & $\begin{array}{l}\mathrm{Z} \\
\mathbf{o} \\
\mathbf{n} \\
\mathbf{e}\end{array}$ & $\begin{array}{l}\text { Clea- } \\
\text { rance } \\
\text { Time } \\
\text { (sec.) }\end{array}$ & $\begin{array}{l}\mathrm{Z} \\
\mathbf{O} \\
\mathbf{n} \\
\mathbf{e}\end{array}$ & $\begin{array}{l}\text { Clea- } \\
\text { rance } \\
\text { Time } \\
\text { (sec.) }\end{array}$ & $\begin{array}{l}\mathrm{Z} \\
\mathbf{o} \\
\mathbf{n} \\
\mathrm{e}\end{array}$ & $\begin{array}{l}\text { Clea- } \\
\text { rance } \\
\text { Time } \\
\text { (sec.) }\end{array}$ \\
\hline 10 & $0^{\circ}$ & $\mathrm{Z1}$ & 0.053 & $\mathrm{Z} 2$ & 0.063 & $\mathrm{Z} 2$ & 0.063 \\
\hline 20 & $0^{\circ}$ & $\mathrm{Z1}$ & 0.053 & $\mathrm{Z} 2$ & 0.063 & $\mathrm{Z} 2$ & 0.063 \\
\hline 30 & $0^{\circ}$ & $\mathrm{Z1}$ & 0.053 & $\mathrm{Z1}$ & 0.053 & $\mathrm{Z} 2$ & 0.063 \\
\hline 40 & $0^{\circ}$ & $\mathrm{Z1}$ & 0.053 & $\mathrm{Z1}$ & 0.053 & $\mathrm{Z} 2$ & 0.063 \\
\hline 50 & $90^{\circ}$ & $\mathrm{Z} 1$ & 0.048 & $\mathrm{Z1}$ & 0.049 & $\mathrm{Z} 2$ & 0.058 \\
\hline 60 & $90^{\circ}$ & $\mathrm{Z} 1$ & 0.048 & $\mathrm{Z1}$ & 0.048 & $\mathrm{Z} 2$ & 0.058 \\
\hline 70 & $90^{\circ}$ & $\mathrm{Z} 1$ & 0.049 & $\mathrm{Z1}$ & 0.049 & $\mathrm{Z} 2$ & 0.059 \\
\hline 80 & $90^{\circ}$ & $\mathrm{Z1}$ & 0.059 & $\mathrm{Z1}$ & 0.050 & $\mathrm{Z} 1$ & 0.059 \\
\hline 90 & $45^{\circ}$ & $\mathrm{Z} 1$ & 0.05 & $\mathrm{Z1}$ & 0.049 & $\mathrm{Z} 1$ & 0.050 \\
\hline 100 & $45^{\circ}$ & $\mathrm{Z1}$ & 0.051 & $\mathrm{Z1}$ & 0.050 & $\mathrm{Z} 1$ & 0.050 \\
\hline $\begin{array}{c}\text { aver- } \\
\text { age }\end{array}$ & & & 0.0517 & & 0.0527 & & 0.0586 \\
\hline
\end{tabular}


Table 2 shows the clearance time for the studied three ended line using Agent technology. The same situation concerning fault locations, fault inception angles and fault resistance is considered.

It should be noted that an opening time of approximately three cycles for the circuit breaker utilized was considered. It isolates the fault by interrupting the current at or near a current zero. A coordination delay for zone two of the order of $0.3 \mathrm{~s}$ was also utilized.

As mentioned before, in the proposed technology, when a fault is detected in the first zone of any of the relays located at the three terminals, a trip signal is sent through the communication network to the other two ends in order to isolate the fault. A transfer trip time of $10 \mathrm{~ms}$ is considered.

A considerable decrease in clearance time considering the proposed scheme can be observed. This scheme is similar to a pilot relaying system utilizing a dedicated communication channel to produce tripping at all other terminals.

\section{CONCLUSION}

An application of agents acting within a communication structure to adaptive relay settings for multi-terminal lines was presented in this paper. Results illustrating the performance of the new method proposed compared to conventional fixed settings were shown. The new approach based on agents was divided in an adaptive pre-fault approach and a post-fault transfer trip action. In order to test the proposed scheme, a digital simulation of a faulted transmission line was performed utilizing the PSCAD/EMTDC software. The protection system associated with this configuration was also simulated both for conventional non-pilot protection as well as for the Agent based relaying. The results showed that the new approach would alter adaptive settings to ensure correct performance over a wide variety of operation conditions, which is an improvement compared to fixed settings for the relays. Moreover, a considerable decrease in clearance time compared to conventional non-pilot protection was observed. It should then be emphasized that the new approach is not only a solution for a well-known problem, but also a very attractive alternative to improve the performance of protection systems in the new millennium.

\section{VII . ACKNOWLEDGMENTS}

The authors acknowledge the research support by subcontract No. 35352-6085 with Cornell University under WO 8333-04 from the Electric Power Institute and the U.S. Army Research Office as well as FAPESP (Fundação de Amparo à Pesquisa do Estado de São Paulo -Brazil).

\section{REFERENCES}

[1] S.H. Horowitz and A.G. Phadke, Power System Relaying, John Wiley and Sons, 1996

[2] IEEE Working Group, "Protection Aspects of Multi-terminal Lines", IEEE Special Publication, 79 TH0056-2-PWR, 1979.

[3] G.D. Rockefeller, C.L. Wagner, J.R. Linders, K.L. Hicks and D.T. Rizy, "Adaptive Transmission Relaying Concepts for Improved Performance", IEEE Transactions on Power Delivery, Vol.3, nº 4, October 1988, pp.1446-58.

[4] S.H. Horowitz, A.G. Phadke and J.S. Thorp, "Adaptive Transmission System Relaying", IEEE Transactions on Power Delivery, Vol.3, $\mathrm{n}^{\circ} 4$, October 1988, pp.1436-45.

[5] Y.Q. Xia, K.K. Li and A.K. David, "Adaptive Relay Setting for Stand Alone Digital Distance Protection", IEEE Transactions on Power Delivery, Vol.9, n ${ }^{\circ}$ 1, January 1994, pp. 480- 91.

[6] Y.Q. Xia, A.K. David and K.K. Li, "High-Resistance Faults on a MultiTerminal Line: Analysis, Simulated Studies and an Adaptive Distance Relaying Scheme", IEEE Transactions on Power Delivery, Vol.9, $\mathrm{n}^{\circ} 1$, January 1994, pp. 492- 500.

[7] B. Stedall, P. Moore, A. Johns, J. Goody and M. Burt "An Investigation into the use of Adaptive Setting Techniques for Improved Distance Backup Protection”, IEEE Transactions on Power Delivery, Vol.11, n ${ }^{\circ}$, April 1996, pp. 757- 62.

[8] Y. Tomita, C. Fukui, H. Kudo, J. Koda, K. Yabe, "A Cooperative Protection System with an Agent Model", IEEE Transactions on Power Delivery, Vol.13, nº 4, October 1998, pp. 1060- 66.

[9] M. Genesereth and S. Ketchpel, "Software Agents", Communications of the ACM, Vol. 37, n. 7, July 1994, pp. 48-52, 147.

[10] C. Heckman and J.O. Wobbrock, "Liability for Autonomous Agent Design", Autonomous Agents 98, pp. 392-399.

[11] F. Schneider, "Towards Fault-tolerant and Secure Agentry", Proceedings $111^{\text {th }}$ International Workshop on Distributed Algorithms, Lecture Notes in Computer Science, Volume 1320, September 1997, pp. 1-14.

[12] K. Birman, M. Hayden, O. Ozkasap, Z. Xiao, M. Budiu and Y. Minsky "Bimodal Multicast" ACM Transactions on Computer Systems, Vol. 17, No. 2, May 1999.

[13] PSCAD/EMTDC Manual, Getting Started, Manitoba HVDC Center 1998

[14] A. Phadke and J. Thorp, Computer Relaying for Power Systems, John Wiley and Sons INC., 1988. 


\section{BIOGRAPHIES}

Denis V. Coury was born in Brazil, in 1960. He received a B.Sc. degree in Electrical Engineering from the Federal University of Uberlandia, Brazil in 1983, a MSc degree from the University of São Paulo, Brazil in 1986 and a Ph.D. degree from Bath University, England in 1992. He joined the Department of Electrical Engineering, University of São Paulo, São Carlos, Brazil in 1986, where he is an Associate Professor in the Power Systems Group. Presently he is at Cornell University for his Sabbatical leave. His areas of research interest are Power System Protection as well as new techniques for Power System Control and Protection including the use of Expert Systems and Artificial Neural Networks. Email coury@ee.cornell.edu

James S. Thorp is the Charles N. Mellowes Professor in Engineering and Director of the School of Electrical Engineering at Cornell University. In 1976, he was a faculty intern at the AEP Service Corporation. He was an associate editor for IEEE Transactions on Circuits and Systems from 1985 to 1987. In 1988, he was an overseas fellow at Churchill College, Cambridge, England. He is a member of the National Academy of Engineering, a Fellow of IEEE and a member of the IEEE Power System Relaying Committee, CIGRE, Eta Kappa Nu, Tau Beta Pi and Sigma Xi. Email jst6@ cornell.edu

Kenneth M. Hopkinson is a native of Charlotte, North Carolina. He received his BS degree in Computer Science from Rensselaer Polytechnic Institute in 1997. Since that time, Ken has been working towards his Ph.D. degree in Computer Science at Cornell University in Kenneth Birman's Ensemble research group. His areas of interest include distributed computer systems and network communications protocols. Ken Hopkinson is currently investigating communications issues arising in the power grid under deregulation. Email hopkik@cs.cornell.edu

Kenneth P. Birman is a Professor of Computer Science at Cornell University. A Fellow of the ACM, Birman has published extensively on reliable, secure distributed computing since joining Cornell in 1982. He developed the Isis Toolkit, which controls communication in the New York Stock and Swiss Exchanges, the French air traffic control system, and oversaw development of Cornell's Horus, Ensemble and Spinglass systems. He was Editor in Chief of ACM Transactions on Computer Systems from 1994-1999. He has advised the government on hardening the nationally critical communications infrastructure, and was co-chair of the 1995 DARPA ISAT study that set the research agenda in this field. Email ken@cs.cornell.edu 